University of Wollongong

Research Online

Australian Institute for Innovative Materials -

Papers

Australian Institute for Innovative Materials

$1-1-2016$

\title{
Shape-engineerable composite fibers and their supercapacitor application
}

Kang Min Kim

Hanyang University

Jae Ah Lee

Hanyang University

Hyeon Jun Sim

Hanyang University

Kyung Ah Kim

Chungbuk National University

Rouhollah Jalili

University of Wollongong, rjalili@uow.edu.au

See next page for additional authors

Follow this and additional works at: https://ro.uow.edu.au/aiimpapers

Part of the Engineering Commons, and the Physical Sciences and Mathematics Commons

Research Online is the open access institutional repository for the University of Wollongong. For further information contact the UOW Library: research-pubs@uow.edu.au 


\title{
Shape-engineerable composite fibers and their supercapacitor application
}

\author{
Abstract \\ Due to excellent electrical and mechanical properties of carbon nano materials, it is of great interest to \\ fabricate flexible, high conductive, and shape engineered carbon based fibers. As part of these \\ approaches, hollow, twist, ribbon, and other various shapes of carbon based fibers have been researched \\ for various functionality and application. In this paper, we suggest simple and effective method to control \\ the fiber shape. We fabricate the three different shapes of hollow, twisted, and ribbon shaped fibers from \\ wet spun giant graphene oxide (GGO)/single walled-nanotubes (SWNTs)/poly(vinyl alcohol) (PVA) gels. \\ Each shaped fibers exhibit different mechanical properties. The average specific strengthes of the hollow, \\ twist, and ribbon fibers presented here are 126.5, 106.9, and 38.0 MPa while strain are 9.3, 13.5, and 5\%, \\ respectively. Especially, the ribbon fiber shows high electrical conductivity (524 $\pm 64 \mathrm{~S} \mathrm{~cm}-1)$ and areal \\ capacitance $(2.38 \mathrm{mF} \mathrm{cm}-2)$. \\ Disciplines \\ Engineering | Physical Sciences and Mathematics \\ Publication Details \\ Kim, K., Lee, J. Ah., Sim, H. Jun., Kim, K., Jalili, R., Spinks, G. M. \& Kim, S. Jeong. (2016). Shape- \\ engineerable composite fibers and their supercapacitor application. Nanoscale, 8 (4), 1910-1914.

\section{Authors} \\ Kang Min Kim, Jae Ah Lee, Hyeon Jun Sim, Kyung Ah Kim, Rouhollah Jalili, Geoffrey M. Spinks, and Seon \\ Jeong Kim
}




\section{Nanoscale}

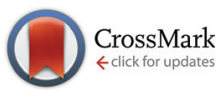

Cite this: Nanoscale, 2016, 8, 1910

Received 15th October 2015,

Accepted 6th January 2016

DOI: $10.1039 / \mathrm{c} 5 \mathrm{nr} 07147 \mathrm{j}$

www.rsc.org/nanoscale

\section{Shape-engineerable composite fibers and their supercapacitor application $\dagger$}

\author{
Kang Min Kim, ${ }^{a}$ Jae Ah Lee, ${ }^{a, b}$ Hyeon Jun Sim, ${ }^{a}$ Kyung-Ah Kim, ${ }^{* c}$ Rouhollah Jalili, ${ }^{d}$ \\ Geoffrey M. Spinks ${ }^{d}$ and Seon Jeong Kim*a
}

Due to excellent electrical and mechanical properties of carbon nano materials, it is of great interest to fabricate flexible, high conductive, and shape engineered carbon based fibers. As part of these approaches, hollow, twist, ribbon, and other various shapes of carbon based fibers have been researched for various functionality and application. In this paper, we suggest simple and effective method to control the fiber shape. We fabricate the three different shapes of hollow, twisted, and ribbon shaped fibers from wet spun giant graphene oxide (GGO)/single walled-nanotubes (SWNTs)/ poly(vinyl alcohol) (PVA) gels. Each shaped fibers exhibit different mechanical properties. The average specific strengthes of the hollow, twist, and ribbon fibers presented here are 126.5, 106.9, and $38.0 \mathrm{MPa}$ while strain are $9.3,13.5$, and $5 \%$, respectively. Especially, the ribbon fiber shows high electrical conductivity $\left(524 \pm 64 \mathrm{~S} \mathrm{~cm}^{-1}\right)$ and areal capacitance $\left(2.38 \mathrm{mF} \mathrm{cm}^{-2}\right)$.

Electrically conductive, durable, and flexible fibers can be used for the wearable textile industry. Various approaches have been reported for fabrication of diverse shapes of fibers based on conducting polymers, graphene oxide (GO), carbon nanotubes (CNTs), and their composites for various applications. Each form has unique functionality and range of applications; e.g., our previous research shows a flexible yarn supercapacitor having high energy density and power capability by biscrolling a poly(3,4-ethylenedioxythiophene) (PEDOT)/CNT nanomembrane for textile and microdevice applications. ${ }^{1}$ Wet-spun GO fiber with helical arrangement of graphene sheets by twisting shows moisture-driven rotational motor. ${ }^{2}$ The fabrication of tubular structures has been recently achieved by electro-

\footnotetext{
${ }^{a}$ Center for Self-powered Actuation and Department of Biomedical Engineering, Hanyang University, Seoul 04763, South Korea. E-mail: sjk@hanyang.ac.kr

${ }^{b}$ The Alan G. MacDiarmid NanoTech Institute, University of Texas at Dallas, Richardson, TX. 75083, USA

${ }^{c}$ Dept. of Biomedical Engineering, School of Medicine, Chungbuk National University, Cheongju 28644, Korea.E-mail: kimka@chungbuk.ac.kr

${ }^{d}$ Intelligent Polymer Research Institute, ARC Centre of Excellence for Electromaterials Science, University of Wollongong, Wollongong, NSW 2522, Australia

$\dagger$ Electronic supplementary information (ESI) available. See DOI: 10.1039/ c5nr07147j
}

spinning and these tubular structures give hope for stimulusresponsive devices and self-powered micromotors for fluidics, catalysis, purification, and separation. ${ }^{3,4}$ Here, we focus on a simple and effective fabricating method of controlling fiber shapes that presents benefits for various applications.

Graphene and CNTs are attractive materials because of their excellent electrical, thermal, and mechanical properties. $^{5-10}$ Recently, giant graphene oxide (GGO) sheets with extremely high aspect ratios are being used as building blocks because GGO has affinity with water and high-performance mechanical strength and electrical conductivity. ${ }^{11,12}$ In addition, remarkably improved mechanical and electrical properties of graphene and CNTs composite fibers according to the ratios of graphene and CNT were demonstrated. ${ }^{13,14}$

Here we report that a simple method during the fabrication process could induce three different shapes in wet-spun GGO/ single-walled nanotubes (SWNTs)/poly(vinyl alcohol) (PVA) gels: hollow fibers, twisted fibers, and ribbons. Each shape had different porosities, and different mechanical and electrical properties. Although the specific strength of various shapes obtained from spun GGO/SWNT/PVA ribbon gels are similar to other wet-spun fibers consisting of SWNT, GO, reduced graphene, or their composites, the wrinkled structures and the sliding effect of the lamellae along the layer of large-size graphene help in improving its specific strain properties. Importantly, we also demonstrate the high-capacitance supercapacitor application of the polymer-free GGO/SWNT ribbon, which has a high surface area to volume ratio among the various shapes.

The fabrication process for fibers based on GGO and SWNT is shown in Fig. 1. It is interesting to note that the three different shapes and inner structures of fibers can be controlled during the fabrication process. This process occurs in two steps following spinning and evaporation. In the first step, a well-dispersed aqueous solution, containing $0.15 \mathrm{wt} \%$ of each GGO and SWNTs, and 0.5 wt $\%$ of sodium dodecylbenzene sulfonate (SDBS) were prepared for fiber spinning. The dispersions were injected through the syringe into the slowly rotated coagulation bath of $5 \mathrm{wt} \%$ PVA dissolved in deionized 


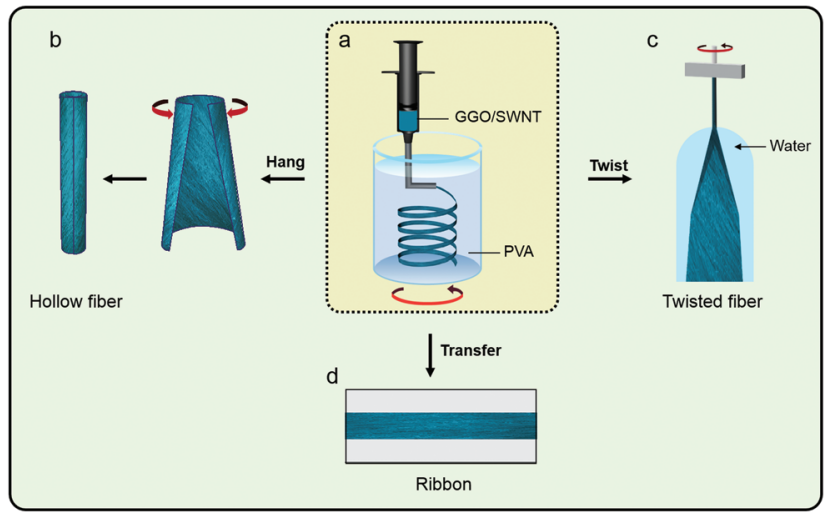

Fig. 1 Shape-engineerable fabrication process based on wet-spun GGO/SWNT/PVA ribbon gels. (a) Schematic illustration of the wet-spinning setup. $(b-d)$ Schematic illustrations of the shape-engineerable fibers according to the various evaporation processes, i.e., hanging, twisting, and transferring, respectively.

(DI) water. This process leads to the formation of ribbons that keep stable in the fluid. The formation mechanism of the ribbon fiber can be explained by two main factors as mentioned in the literatures. ${ }^{15,16}$ First is high viscosity of the polymer coagulation bath because the large viscosity difference between polymer and injected GGO/SWNT solution can induce sheer stress that allows GGO sheets to be rapidly stuck together when they are coming out the tip, resulting in ribbon shape. Second, electrostatic interaction between used PVA polymer and GGO sheet in our experiment is good enough to lead ribbon shape formation of the wet-spun fiber. The GGO/ SWNT gel rises to the surface of the PVA solution just after spinning. Finally, the GGO/SWNT/PVA hybrid ribbon gel was formed and sank to the bottom of the coagulation bath after 18 to 24 hours in the oven at $60^{\circ} \mathrm{C}$.

After washing in DI water more than ten times, in the second step, three different shapes and structures could be obtained according to various evaporation methods. At our first attempt, the as-spun gels were simply hung out of the DI water as described in Fig. 1b. When the as-spun gel passed through a DI water/air interface, it started to scroll from both lateral edges towards the center to form a hollow fiber. The asspun gels were twisted (Fig. 1c). One end of the as-spun gel was attached on the shaft of an electric motor and the gel was slowly twisted to induce 10000-12 000 turns per meter in the water. Finally, we obtained a highly densified GGO/SWNT/PVA twisted fiber. The GGO/SWNT/PVA ribbon was fabricated by a simple transfer method in the water. The as-spun gels were transferred on the polytetrafluoroethylene (PTFE) film (see Fig. 1d); next, pentane was flowed over the transferred ribbon several times. After drying, the ribbon could be stripped from the PTFE film.

The scanning electron microscope (SEM) images (Fig. 2) show the shape-engineeralbe fibers (hollow fiber, twisted fiber, and ribbon) fabricated during the spinning and evaporation processes. All samples were thermally annealed at $600{ }^{\circ} \mathrm{C}$
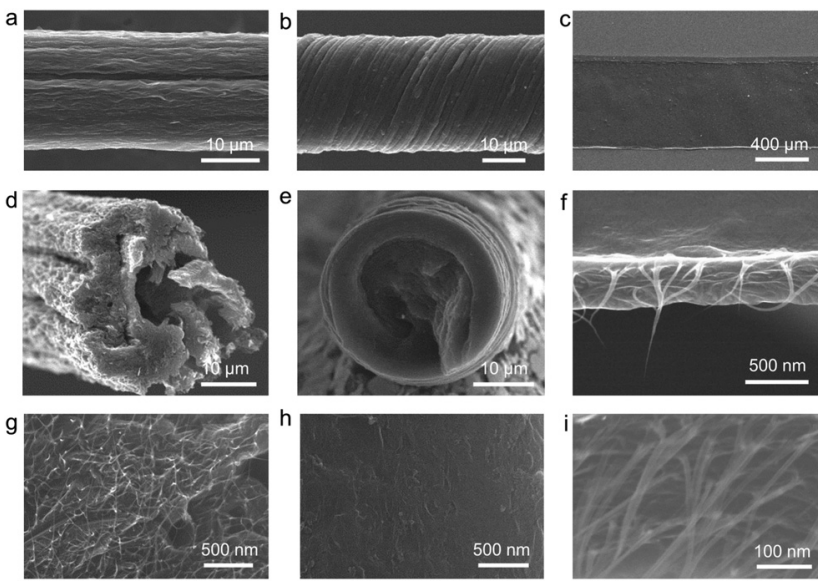

Fig. 2 SEM images of surface and cross-sections. (a, d, g) Hollow fibers, $(b, e, h)$ twisted fibers, and $(c, f, i)$ ribbons. All samples were annealed at $600{ }^{\circ} \mathrm{C}$ for $2 \mathrm{~h}$ under $\mathrm{Ar} / \mathrm{H}_{2}$ (95/5 vol\%) gas mixture.

under $\mathrm{Ar} / \mathrm{H}_{2}(95 / 5$ vol\%) gas mixture for $2 \mathrm{~h}$ to remove the PVA. The surface and cross-section SEM images of the GGO/SWNT hollow fiber (o.d. $\sim 34 \mu \mathrm{m}$ and outer wall thickness $\sim 12 \mu \mathrm{m}$ ) are observed in Fig. 2a, d and g. Surface tension occurred during evaporation; water results in largely decreasing the diameter and making partially oriented wrinkles on the surface. The surface and cross-section images of twisted GGO/SWNT fiber (Fig. 2b, e and h) were clearly different from those of hollow fiber. The twisted fiber had a diameter of $\sim 28 \mu \mathrm{m}$ and a steep slope of the bias angle, $\alpha\left(\sim 66^{\circ}\right)$, which means that the GGO/SWNT spun-gel could endure the high torsional force during twisting. Twisting generates high compressive forces, resulting in a compact structure of GGO/SWNT (Fig. 2e and h). The GGO/SWNT twisted fiber showed a relatively smooth surface and a circular cross section. SEM images of a ribbon fabricated by the simple transfer method are shown in Fig. 2c, $\mathrm{f}$ and $\mathrm{i}$. The ribbon width (500 to $700 \mu \mathrm{m})$ scarcely showed any changes before and after evaporation. The expected application for a ribbon with a high surface-area-to-volume ratio is a supercapacitor for energy storage devices.

To measure the mechanical robustness of the shape- engineerable composite fibers, we performed tensile tests using a universal testing machine. Fig. 3 shows the stress-strain curves of GGO/SWNT/PVA hybrid hollow fiber, twisted fiber, and ribbon. The specific strength and strain values of the hollow fiber were $126.5 \pm 22.5 \mathrm{MPa}$ and $9.3 \pm 1.2 \%$, respectively (Table 1). Although this mechanical strength was similar to that of wet-spun SWNT, GO, reduced graphene, and their composite-based fibers (80 to $450 \mathrm{MPa}$ ) ${ }^{11,15,19-21}$ the strain of hollow fibers surpassed previous wet-spun fibers $(2.8 \%$ to $6.8 \%)$. This mechanical strain was $\sim 3.3$ times higher than that of wet-spun GOs hollow fiber prepared by the coaxial two-capillary spinning strategy. ${ }^{22}$ The twisted fiber and ribbon showed lower mechanical strength than hollow fibers (106.9 and $38.0 \pm$ 1.3 MPa, respectively). The shape-engineerable fibers having different wrinkled structures were formed by different drying 


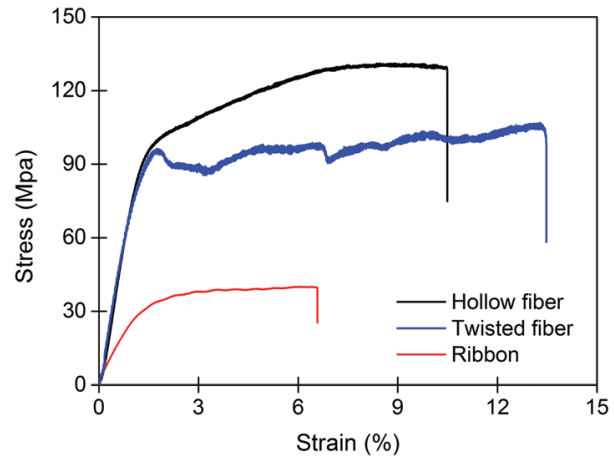

Fig. 3 Mechanical properties of various types of fiber from wet-spun GGO/SWNT/PVA.

conditions, resulting in a variety of mechanical properties. The strains of twisted fiber and ribbon showed $13.5 \%$ and $5.0 \% \pm$ $1.0 \%$, respectively. The sliding of lamellae along the layer of giant graphene could explain the large physical strain. ${ }^{11}$ Table 1 summarizes the mechanical properties compared to previous reports on wet-spun fibers consisting of SWNT, GO, reduced graphene, and their composites.

The X-ray diffraction (XRD) patterns of the GGO/SWNT ribbons before and after annealing are shown in Fig. 4. The dried GGO/SWNT ribbons showed sharp diffraction peaks at the 2 theta positions of $26.46^{\circ}$ and $30.72^{\circ}$, corresponding to the interlayer spacings of 3.37 and $2.91 \AA$, respectively. XRD analysis of the GGO/SWNT ribbon annealed at $600{ }^{\circ} \mathrm{C}$ for $2 \mathrm{~h}$ displayed a different XRD pattern with $d$-spacings of 3.35 and 2.71 $\AA$. The peak shift in increase direction of diffraction angle means that the $d$-spacing value is reduced, ${ }^{17,18}$ which means $d$-space our ribbon GGO/CNT wet-spun fiber decrease. This explains the water and other unnecessary chemical functionalities in the GGO sheets were effectively removed by heat treatment, indicating the effectiveness of the reduction process. ${ }^{17,18}$

Because the ribbon had the highest surface-area-to-volume ratio among the three shapes, we evaluated its electrochemical properties. It also had a high electrical conductivity value of $524 \pm 64 \mathrm{~S} \mathrm{~cm}^{-1}$ after annealing at $600 \mathrm{C}$ for $2 \mathrm{~h}$ under Ar gas. The electrochemical capacitance for the reduced GGO/SWNT

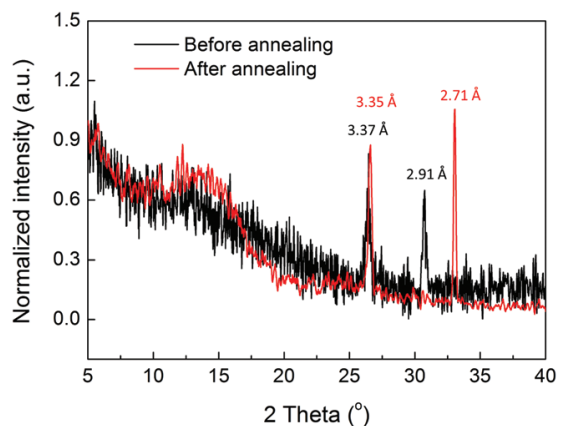

Fig. 4 XRD characterization. Comparison of XRD patterns of the ribbon before (black) and after (red) annealing at $600{ }^{\circ} \mathrm{C}$ for $2 \mathrm{~h}$ under $\mathrm{Ar} / \mathrm{H}_{2}$ (95/5 vol\%) gas mixture.

ribbon was determined in three- and two-electrode system using $0.1 \mathrm{M} \mathrm{Na}_{2} \mathrm{SO}_{4}$ liquid and PVA-LiCl solid electrolytes, respectively. Cyclic voltammetry (CV) curves for different voltage scan rates are presented in Fig. S2 $\dagger$ (three-electrode system) and Fig. 5a (two-electrode system). The areal and length specific capacitances were about $\sim 2.38 \mathrm{mF} \mathrm{cm}^{-2}$ and $\sim 0.118 \mathrm{mF} \mathrm{cm} \mathrm{cm}^{-1}$, respectively, as shown in Fig. 5b. The areal and length capacitances of the ribbon were obtained using $\left(I_{\mathrm{a}}+\left|I_{\mathrm{c}}\right|\right) t /(2 A E)$, where $I_{\mathrm{a}}$ and $I_{\mathrm{c}}$ are the average anodic and cathodic voltammetry current on the anodic and cathodic scans, $t$ is scan time, $A$ is the surface area of the active material, and $E$ was the potential range of the $\mathrm{CV}$ curves. At the high scan rate of $20 \mathrm{~V} \mathrm{~s}^{-1}$, the areal capacitance was $\sim 0.1 \mathrm{mF} \mathrm{cm} \mathrm{cm}^{-2}$, which means that the reduced GGO/SWNT ribbons were able to show their moderate performance. The areal capacitance measured at $10 \mathrm{mV} \mathrm{s}^{-1}$ was similar to that of the stacked multilayers of reduced $\mathrm{GO} / \mathrm{CNT}\left(2.6 \mathrm{mF} \mathrm{cm}{ }^{-2}\right){ }^{23}$ Also, the areal capacitance of the GGO/SWNT ribbon was much higher than that of a typical double-layer capacitance with a smooth surface $\left(10-20 \mathrm{uF} \mathrm{cm}^{-2}\right) .{ }^{24}$ The galvanostatic charge-discharge curves of reduced GGO/SWNT ribbon at different current density of 30,60 and $100 \mathrm{uA} \mathrm{cm}^{-2}$ are shown in Fig. 5c. Moreover, as shown in Fig. 5d, the reduced GGO/SWNT ribbon shows excellent capacitance retention performance $( \pm 4 \%$ capacitance deviation) during 6000 charge-discharge cycles at $200 \mathrm{mV} \mathrm{s}^{-1}$.

Table 1 Mechanical properties of hollow fibers, twisted yarns, and ribbon consisting of GGO/SWNT/PVA prepared by our shape-engineerable method and previous reports

\begin{tabular}{|c|c|c|c|c|}
\hline Materials and shape (coagulation bath) & Young's modulus [GPa] & Tensile stress $[\mathrm{MPa}]$ & Tensile strain $[\%]$ & Ref. \\
\hline GGO/SWNT/PVA twisted yarn (PVA) & 8.8 & 106.9 & 13.5 & This work \\
\hline SWNT/PVA fiber (PVA) & 15 & 150 & 3 & 13 \\
\hline GO fiber $(\mathrm{NaOH} /$ methanol) & 5.4 & 102 & 6.8 & 17 \\
\hline rGO/PVA fiber (PVA) & 9.9 & 161 & 3.0 & 18 \\
\hline rGO/SWNT fiber (water) & NA & 84 & 3.3 & 12 \\
\hline Graphene ribbon (chitosan) & - & 34.2 & 14.4 & 14 \\
\hline
\end{tabular}


a

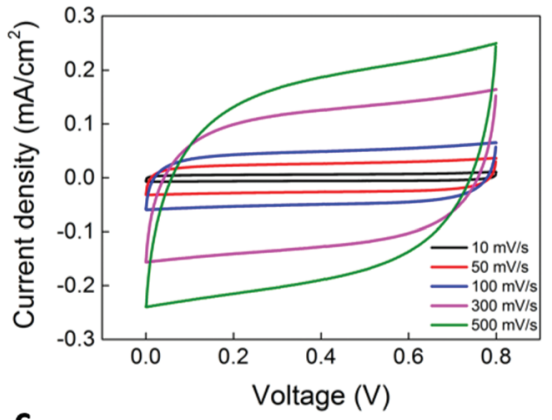

C

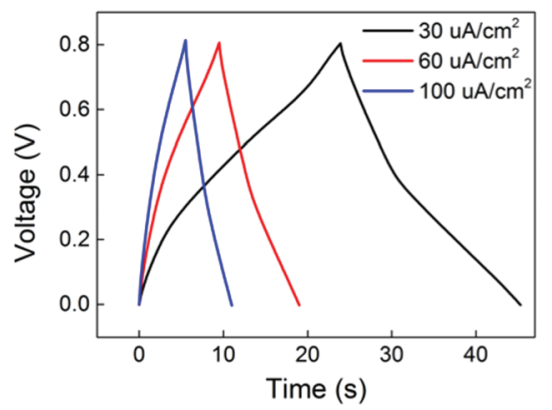

b

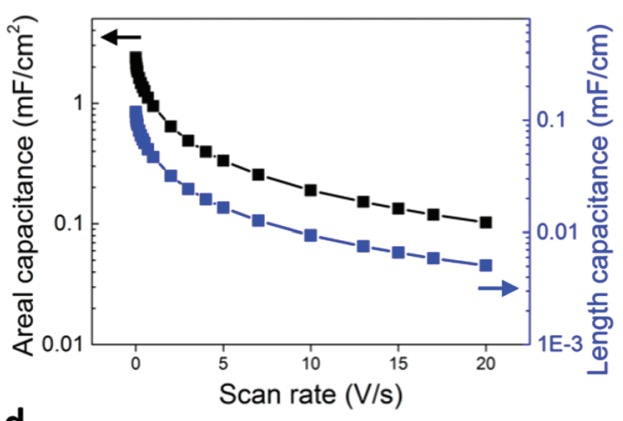

d

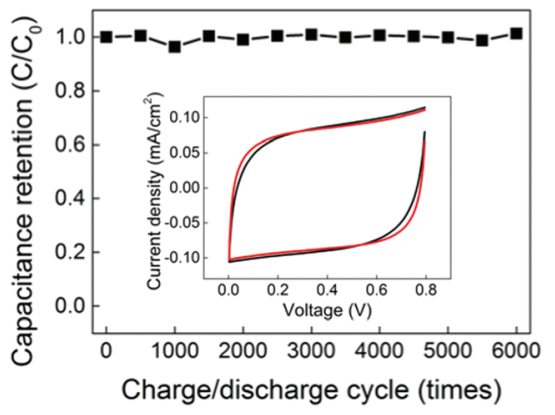

Fig. 5 Electrochemical performances of the reduced GGO/SWNT ribbon are measured in two electrode system using PVA-LiCl gel electrolyte. (a) $\mathrm{CV}$ curves measured at scan rates from 10 to $500 \mathrm{mV} \mathrm{s}^{-1}$ and (b) calculated areal and length capacitance retention versus various scan rates up to $20 \mathrm{~V} \mathrm{~s}^{-1}$. (c) Galvanostatic charge-discharge curves. (d) Capacitance retention is measured under repeated CV cycles at $200 \mathrm{mV} \mathrm{s}$. The inset in (d) compares CV curves first and $6000^{\text {th }} \mathrm{CV}$ area.

\section{Conclusions}

In summary, we provide a new simple method to fabricate various fiber shapes such as hollow fibers, twisted fibers, and ribbons from wet-spun GGO/SWNT/PVA ribbon gels. The shape-engineerable composite fibers differed in density and surface morphology, which resulted in different mechanical and electrical properties. We observed that wrinkled structures formed along a fiber had improved tensile strain values. The helical arrangement of graphene sheets which obtained by twisting induced more elongation than other arrangements. We also demonstrated a supercapacitor with high capacitance from the polymer-free GGO/SWNT ribbon attached to a current collector such as glassy carbon. We believe that shape-engineerable fiber during the spinning and evaporation processes present numerous advantages and have application potential in various fields.

\section{Acknowledgements}

This work was supported by the Creative Research Initiative Center for Self-powered Actuation of the Ministry of Science, ICT and Future Planning and the KOREA-US Air Force Cooperation Program (Grant No.2013K1A3A1A32035592) in Korea; Air Force Grant AOARD-13-4119, Air Force Office of Scientific Research grant FA9550-12-1-0211, and Robert A. Welch Foundation grant AT-0029 in the USA; and the Austra- lian Research Council through the Centre of Excellence and Professorial Fellowship Programs. Also, K. M. Kim and J. A. Lee contributed equally to this work.

\section{Notes and references}

1 J. A. Lee, M. K. Shin, S. H. Kim, H. U. Cho, G. M. Spinks, G. G. Wallace, M. D. Lima, X. Lepró, M. E. Kozlov, R. H. Baughman and S. J. Kim, Nat. Commun., 2013, 4, 1970.

2 H. Cheng, Y. Hu, F. Zhao, Z. Dong, Y. Wang, N. Chen, Z. Zhang and L. Qu, Adv. Mater., 2014, 26, 2909.

3 D. Li, J. T. McCann and Y. Xia, Small, 2005, 1, 83.

4 Y. Zhao, X. Cao and L. Jiang, J. Am. Chem. Soc., 2007, 129, 764.

5 B. E. Conway, Electrochemical supercapacitors: scientific fundamentals and technological applications, Kluwer Academic/ Plenum Publishers, New York, 1999.

6 P. Simon and Y. Gogotsi, Nat. Mater., 2010, 9, 146.

7 X. Sun, H. Sun, H. Li and H. Peng, Adv. Mater., 2013, 25, 5153.

8 H. Cheng, C. Hu, Y. Zhao and L. Qu, NPG Asia Mater., 2014, 6, e113.

9 X. Feng, Z. Yan, N. Chen, Yu Zhang, Y. Ma, X. Liu, Q. Fan, L. Wang and W. Huang, J. Mater. Chem. A, 2013, 1, 12818.

10 X. Feng, N. Chen, Yu Zhang, Z. Yan, X. Liu, Y. Ma, Q. Shen, L. Wang and W. Huang, J. Mater. Chem. A, 2014, 2, 9178. 
11 R. Jalili, S. H. Aboutalebi, D. E. Lzadeh, R. L. Shepherd, J. Chen, S. Aminorroaya-Yamini, K. Konstantinov, A. I. Minett, J. M. Razal and G. G. Wallace, Adv. Funct. Mater., 2013, 23, 5345.

12 Z. Xu, H. Sun, X. Zhao and C. Gao, Adv. Mater., 2013, 25, 188.

13 M. K. Shin, B. Lee, S. H. Kim, J. A. Lee, G. M. Spinks, S. Gambhir, G. G. Wallace, M. E. Kozlov, R. H. Baughman and S. J. Kim, Nat. Commun., 2012, 3, 650.

14 D. Yu, K. Goh, H. Wang, L. Wei, W. Jiang, Q. Zhang, L. Dai and Y. Chen, Nat. Nanotechnol., 2014, 9, 555.

15 B. Vigolo, A. Penicaud, C. Coulon, C. Sauder, R. Pailler, C. Journet, P. Bernier and P. Poulin, Science, 2000, 290, 1331.

16 J. Sun, Y. Li, Q. Peng, S. Hou, D. Zou, Y. Shang, Y. Li, P. Li, Q. Du, Z. Wang, Y. Xia, L. Xia, X. Li and A. Cao, ACS Nano, 2013, 7, 10225.
17 S. H. Aboutalebi, A. T. Chidembo, M. Salari, K. Konstantinov, D. Wexler, H. K. Liu and S. X. Dou, Energy Environ. Sci., 2011, 4, 1855.

18 S. H. Aboutalebi, M. M. Gudarzi, Q. B. Zheng and J.-K. Kim, Adv. Funct. Mater., 2011, 21, 2978.

19 Z. Xu and C. Gao, ACS Nano, 2011, 5, 2908.

20 L. Kou and C. Gao, Nanoscale, 2013, 5, 4370.

21 D. Yu, K. Goh, H. Wang, L. Wei, W. Jiang, Q. Zhang, L. Dai and Y. Chen, Nat. Nanotechnol., 2014, 9, 555.

22 Y. Zhao, C. Jiang, C. Hu, Z. Dong, J. Xue, Y. Meng, N. Zheng, P. Chen and L. Qu, ACS Nano, 2013, 7, 2406.

23 G. D. Moon, J. B. Joo and Y. Yin, Nanoscale, 2013, 5, 11577.

24 S. L. Candelaria, Y. Shao, W. Zhou, X. Li, J. Xiao, J.-G. Zhang, Y. Wang, J. Liu, J. Li and G. Cao, Nano Energy, 2012, 1, 195. 